\title{
Impact of Drought, Heat, and Their Combination on Chlorophyll Fluorescence and Yield of Wild Barley (Hordeum spontaneum)
}

\author{
Christoph Jedmowski, ${ }^{1}$ Ahmed Ashoub, ${ }^{1,2}$ Osama Momtaz, ${ }^{2}$ and Wolfgang Brüggemann ${ }^{1,3}$ \\ ${ }^{1}$ Department of Ecology, Evolution and Diversity, University of Frankfurt, Max von Laue Strasse 13, 60438 Frankfurt, Germany \\ ${ }^{2}$ Agricultural Genetic Engineering Research Institute (AGERI), ARC, Giza 12619, Egypt \\ ${ }^{3}$ Biodiversity and Climate Research Center, Senckenberganlage 25, 60325 Frankfurt, Germany \\ Correspondence should be addressed to Ahmed Ashoub; ashoub@em.uni-frankfurt.de
}

Received 14 July 2015; Revised 15 October 2015; Accepted 18 October 2015

Academic Editor: Zed Rengel

Copyright (C) 2015 Christoph Jedmowski et al. This is an open access article distributed under the Creative Commons Attribution License, which permits unrestricted use, distribution, and reproduction in any medium, provided the original work is properly cited.

The impact of (long-term) drought acclimation and (short-term) heat stress and their combination on fast chlorophyll fluorescence induction curves (OJIP) and grain yield was tested using pot-grown plants of wild barley (Hordeum spontaneum) originating from Northern Egypt. Concerning agronomic traits, the main effect of drought was decreased biomass accumulation and grain yield, while heat specifically affected floral development. The treatments caused specific inhibitions of photosystem II (PSII) functionality. While heat stressed plants showed a reduction of maximum quantum efficiency of PSII $\left(\varphi P_{0}\right)$, an indication of effects on oxygen evolving complex (OEC) functionality, and the connectivity of PSII units, these features were entirely missing in drought acclimated plants. Drought caused a reduction of the Performance Index $\left(\mathrm{PI}_{\mathrm{abs}}\right)$ and of the relative amplitude of the IP-phase of the OJIP induction curve $\left(\Delta V_{\mathrm{IP}}\right)$. Individuals suffering from a combination of drought and heat showed a better ability to recover photosynthetic electron transport after the relief of stress in comparison to heat stressed plants. However, this improved capacity to recover was not accompanied by an increased grain yield. Thus, we conclude that chlorophyll fluorescence measurements provide valuable physiological data; however, their use in agronomic studies for the prediction of agronomic traits should be done with some precaution.

\section{Introduction}

During the forthcoming decades, global agriculture will have to face dramatic climatic changes $[1,2]$. Water availability will be a limiting factor for agricultural activities in arid and semiarid regions. Even areas that do not undergo water availability problems so far are expected to experience prolonged periods of drought. Moreover, increased atmosphere temperature and heat wave incidences are expected to negatively impact crop yield and food security [3].

Drought and heat are two main factors limiting plant productivity, and in the natural environment these two abiotic stresses often occur simultaneously. Thus, understanding the impact of heat and drought on plant development and productivity is particularly valuable. For our study, we used wild barley (Hordeum spontaneum) the progenitor of domesticated $H$. vulgare L. H. spontaneum is self-pollinating annual grass, interfertile with cultivated barley, and mainly distributed in the Mediterranean and Irano-Turanian regions [4], the "Fertile Crescent," and eastward to Central Asia [5]. It is considered a beneficial source for the inbreeding of tolerance genes against biotic and abiotic stresses into cultivated barley [6].

Suzuki et al. [7] summarized the specific impact of drought and heat on growth and development of cereals. Typical consequences of drought are reduced plant height, a decrease of the total number of spikes, and a reduced seed weight, whereas heat stress (HS) affects pollen development, resulting in a lowered number of seeds per plant [8]. In addition, drought and heat also have a specific impact on 
photosynthesis, a key process of plant metabolism. Reduced water availability triggers stomatal closure, minimizing water loss via transpiration and, on the other hand, leading to a lowered $\mathrm{CO}_{2}$ partial pressure inside the leaf. In addition to this stomatal limitation of photosynthesis, nonstomatal effects (e.g., reduced activities of Calvin Benson cycle enzymes) occur under more severe drought stress conditions [9]. Mild HS (approx. $35^{\circ} \mathrm{C}$ ) affects photosynthesis by a decrease of Ribulose 1,5-bisphosphate carboxylase/oxygenase (Rubisco) activity [10], caused by the heat susceptibility of Rubisco activase [11]. At higher temperatures, HS inhibits the photosynthetic electron transport by disturbing photosystem II (PSII) functionality. In particular, the structural integrity of the PSII associated oxygen evolving complex (OEC) is affected [12].

Measurements of chlorophyll fluorescence are reliable tools for the detection of disturbances of photosynthesis caused by environmental stresses [13]. The analysis of fast chlorophyll fluorescence induction curves (OJIP) according to Strasser et al. $[14,15]$ provides quick information about the functionality of the chloroplast electron transport chain. It was shown that OJIP induction curves are sensitive to a plethora of biotic and abiotic stresses [16-18].

The aims of this study are to determine the impact of heat, drought, and their combination on the floral development and the photosynthetic performance of wild barley and to evaluate the potential and the limitations of chlorophyll fluorescence measurements in estimating plant productivity under stress conditions.

\section{Materials and Methods}

2.1. Plant Material and Growth Conditions. H. spontaneum from the northern coastal region of Egypt (Wadi Habies $31^{\circ} 24^{\prime} 00^{\prime \prime} \mathrm{N} / 27^{\circ} 03^{\prime \prime} 00 \mathrm{E}$ ) was used in this study. Seeds were germinated on wet filter paper for 2 days. Four seedlings were transferred into a $16 \times 16 \times 20($ depth $) \mathrm{cm}^{3}$ pot filled with $1 \mathrm{~kg}$ of a commercial peat soil (type C700, Stender Erden, Schermbeck, Germany) and defined as one biological replicate. Seedlings were grown in a climate chamber under $16 \mathrm{~h}$ photoperiod at a light intensity of $400 \mu \mathrm{mol} \mathrm{m}^{-2} \mathrm{~s}^{-1}$, temperatures of $26^{\circ} \mathrm{C}$ (light) $/ 24^{\circ} \mathrm{C}$ (dark), and air humidity of $70 \%$. Volumetric soil water content (SWC) was kept at $30 \%$ using a Theta Probe ML2x sensor combined with a HH2 moisture meter (Delta-T Devices Ltd., Cambridge, UK). Control (CTR) plants were kept under the conditions mentioned above throughout the experiment.

2.2. Stress Treatments. Plants subjected to drought treatment were left unwatered at the stage of 2 leaves for three weeks, until soil water potential $(\Psi)$ reached $-2.5 \mathrm{MPa}$, as measured using a Psypro psychrometer (Wescor, Utah, USA). This longterm drought treatment will be termed "drought acclimation" (DA), sensu Lambers et al. [19] to distinguish it from shortterm stress treatments, like the applied heat stress (HS). For HS treatment, plants at the stage of four leaves were transferred into a transparent incubator with temperature control (Certomat H, Sartorius, Göttingen, Germany) under a light
TABLE 1: Definitions and explanation of the JIP-test parameters used in this study. See Strasser et al. [15] for further information about the theory of the JIP-test and the derivation of the parameters.

\begin{tabular}{|c|c|}
\hline & Fluorescence parameters \\
\hline$F_{0}$ & Minimum fluorescence intensity (at $t=10 \mu \mathrm{s}$ ) \\
\hline$F_{M}$ & Maximum fluorescence intensity \\
\hline$V_{J}$ & $\begin{array}{l}\text { Relative variable fluorescence at } 2 \mathrm{~ms} \\
V_{J}=\left(F_{2 \mathrm{~ms}}-F_{0}\right) /\left(F_{M}-F_{0}\right)\end{array}$ \\
\hline$V_{I}$ & $\begin{array}{l}\text { Relative variable fluorescence at } 30 \mathrm{~ms} \\
V_{I}=\left(F_{30 \mathrm{~ms}}-F_{0}\right) /\left(F_{M}-F_{0}\right)\end{array}$ \\
\hline \multirow[t]{2}{*}{$\Delta V_{\text {IP }}$} & $\begin{array}{l}\text { Relative amplitude of the IP-phase of the OJIP } \\
\text { induction curve; } \Delta V_{\mathrm{IP}}=1-V_{I}\end{array}$ \\
\hline & Quantum efficiencies, flux ratios \\
\hline$\varphi P_{0}$ & Quantum yield of the $Q_{A}$ reduction; $\varphi P_{0}=\left(1-F_{0}\right) / F_{M}$ \\
\hline$\Psi_{0}$ & $\begin{array}{l}\text { Probability that a trapped exciton is used for electron } \\
\text { transport beyond } Q_{A} ; \Psi_{0}=1-V_{J}\end{array}$ \\
\hline \multirow[t]{2}{*}{$\mathrm{RC} / \mathrm{ABS}$} & Density of active RCs on absorption basis \\
\hline & Performance Index \\
\hline $\mathrm{PI}_{\mathrm{abs}}$ & $\begin{array}{l}\text { Performance Index on absorption basis } \\
\mathrm{PI}_{\mathrm{abs}}=[\mathrm{RC} / \mathrm{ABS}] \cdot\left[\varphi P_{0} /\left(1-\varphi P_{0}\right)\right] \cdot\left[\Psi_{0} /\left(1-\Psi_{0}\right)\right]\end{array}$ \\
\hline
\end{tabular}

intensity of $200 \mu \mathrm{mol} \mathrm{m} \mathrm{m}^{-2} \mathrm{~s}^{-1}$. Temperature was increased gradually by $1^{\circ} \mathrm{C}$ per $20 \mathrm{~min}$ from $26^{\circ} \mathrm{C}$ until $42^{\circ} \mathrm{C}$ and plants were incubated for $1.5 \mathrm{~h}$ at this temperature in the light and further $0.5 \mathrm{~h}$ in the dark. Leaf temperature was checked using an infrared thermometer. Air humidity was maintained by supplying water containers to the center of the hood. For combined stresses (CS), plants at the end of DA were subjected to the same heat treatment regime but omitting the presence of air humidity supplies. The third leaf of one plant per pot was used to measure relative leaf water content (RWC) as described by Smart and Bingham [20]. Following stress treatments, plants were recovered by rewatering to $5 \%$ SWC in the case of DA and CS or to $30 \%$ SWC for HS and returned to the growing conditions described in the previous section.

2.3. Chlorophyll Fluorescence Measurements. Fast chlorophyll fluorescence induction curves (OJIP) were measured with a Pocket Plant efficiency analyzer (PEA, Hansatech, King's Lynn, UK), following $1 \mathrm{~h}$ of dark adaptation. Measurements were conducted at the end of the DA, HS, and CS treatments and again after $24 \mathrm{~h}$ of recovery. The induction curves were analyzed according to the JIP-test protocol of Strasser et al. $[14,15]$. Selected parameters of the JIP-test were used to evaluate the impact of the applied stresses on the photosynthetic electron transport. The Performance Index on absorption basis $\left(\mathrm{PI}_{\mathrm{abs}}\right)$ is a compounded parameter quantifying the overall functionality of the electron flow through PSII. The calculation of $\mathrm{PI}_{\mathrm{abs}}$ and some information about the definitions of the used JIP-test parameters are presented in Table 1. To visualize the physiological changes induced by the stress treatments, differential normalised induction curves, $\Delta V_{\mathrm{OJ}}$ and $\Delta V_{\mathrm{OK}}$, were calculated from the original OJIP transients as described in Jedmowski et al. [21]. 
2.4. Agronomic Measurements. Plants exposed to DA and CS were rewatered to $5 \%$ SWC $(\Psi=-1 \mathrm{MPa})$ and kept under this water supply until seed development was completed. CTR and HS plants were kept at a SWC of 30\%. The number of spikes, the relative number of empty spikes (spikes without any seed), and the number of seeds per replicate were counted. In addition, seeds were weighed immediately after harvesting and the weight of one thousand seeds was calculated. All plants of a single pot were considered as one biological replicate.

2.5. Statistical Analysis. Ten measurements per treatment were used to calculate the presented (averaged) OJIP curves, and ten biological replicates were used to carry out the yield analysis measurements. One-way analysis of variance (ANOVA) with Tukey's post hoc test was used to assess the significance of the differences among treatments at a significance level of $p<0.05$. Comparison of two datasets was conducted using Student's $t$-test. All datasets shown are presented as the means + standard deviation (SD). Significant changes were marked with one ( $p<0.05)$, two $(p<0.01)$, or three $(p<0.001)$ asterisks.

\section{Results}

3.1. Plant Water Status. DA and CS treatment reduced the RWC of $H$. spontaneum plants significantly to $80.3 \%$ and $73.1 \%$, respectively, in comparison to the CTR group (96.1\%), whereas under HS the RWC remained stable (94.8\%,). The observed difference between DA and CS was not statistically significant $(p=0.27)$.

3.2. Chlorophyll Fluorescence. All treatments had a specific effect on chlorophyll fluorescence parameters. DA resulted in a decrease of $\mathrm{PI}_{\mathrm{abs}}$, which was partly reversed during recovery (Figure $1(\mathrm{a})$ ). In contrast, the maximum quantum yield was only slightly affected by DA treatment (Figure 1(b)). $\mathrm{PI}_{\text {abs }}(-75 \%)$ and $\varphi P_{0}(-13 \%)$ behaved in a similar way to HS and CS plants. However, significant differences between these two groups were observed after recovery, as only CS plants showed increased $\mathrm{PI}_{\text {abs }}$ and $\varphi P_{0}$ values after returning them to standard growing conditions. The fraction of active $\left(Q_{A}\right.$-reducing) reaction centers on absorption base (RC/ABS) was not altered significantly by DA treatment (Figure 1(c)), while HS and CS caused a reduction to $70 \%$ of the CTR group. Again, recovery revealed differences between HS and CS plants. The relative amplitude of the IP-phase of the OJIP transient $\left(\Delta V_{\text {IP }}\right)$ was significantly reduced by DA treatment (Figure $1(\mathrm{~d})$ ). Recovery measurements showed a slight increase of $\Delta V_{\text {IP }}$, but still the mean value was lower than under well-watered conditions. Under HS and CS, $\Delta V_{\mathrm{IP}}$ increased by $15 \%$, and this effect persisted after recovery of the HS plants. In case of the recovered CS plants, $\Delta V_{\text {IP }}$ dropped to $75 \%$ of the CTR value, similar to the DA recovery plants. Drought had no effect on the relative variable fluorescence of the O-J and the O-K phase of the OJIP induction curves (Figures 2(a) and 2(d)). Again, HS and CS showed an identical reaction under stress conditions (Figures 2(b), 2(c), 2(e), and 2(f)), exhibiting a positive maximum of $\Delta V_{\mathrm{O}}$ (at 300-400 $\mu \mathrm{s}$ ) and $\Delta V_{\mathrm{OK}}$ (at 100-200 $\mu \mathrm{s}$ ). These maxima persisted in plants recovering from HS but decreased $\left(\Delta V_{\text {OJ }}\right)$ or disappeared $\left(\Delta V_{\mathrm{OK}}\right)$ in CS plants after recovery.

3.3. Agronomic Measurements. Drought led to a decrease of the number of spikes per replicate (5.6, Figure 3(a)). CTR plants generated 14.3 spikes on average. CS plants behaved in a similar way to DS plants, exhibiting 6.8 spikes per replicate. HS had no effect on the total number of spikes. In contrast, the number (6.1, Figure 3(b)) and the frequency (52\%, Figure $3(c)$ ) of empty spikes were increased compared to the CTR group $(2.7,20.1 \%)$. DA and CS had no significant effect on the total and relative number of empty spikes. The number of seeds per replicate (Figure 4(a)) was reduced under DA (31.7) and CS (25.2) conditions. Under HS, the amount of seeds was also lowered from approximately 80 to 65 per replicate, yet the difference to the CTR plants (87) was not statistically significant. Seed weight was only slightly affected under the applied stresses (Figure 4(b)). Under DA and CS treatment, plants produced smaller seeds (DA: $32.8 \mathrm{~g} / 1000$ seeds; CS: $33.9 \mathrm{~g} / 1000$ seeds) compared to the CTR group ( $38.1 \mathrm{~g})$.

\section{Discussion}

4.1. Chlorophyll Fluorescence. In our study, all applied treatments affected the functionality of PSII electron transport (Figures 1 and 2). The JIP-test was used in several studies aiming for the quantification of the impact of environmental stresses on photosynthesis. Oukarroum et al. [22] and Jedmowski et al. [21] evaluated the functionality of the photosynthetic electron transport chain functionality of $H$. vulgare varieties under drought stress and during recovery. In these studies, the $\mathrm{PI}_{\mathrm{abs}}$ revealed differences between varieties as a function of drought and rewatering. Additionally, it was shown for wild barley genotypes under drought that the $\mathrm{PI}_{\mathrm{abs}}-$ measured on dark adapted leaves - correlates well with maximum photosynthesis rates measured under saturating light conditions [18]. Thus, the lowered $\mathrm{PI}_{\mathrm{abs}}$ observed in our study supports the theory that the reduced number of spikes and seeds per replicate under DA and CS conditions was caused by reduced carbon assimilation. Drought only had a minor effect on the maximum quantum efficiency of PSII. However, the observed change was statistically significant due to the very small standard deviation of $\varphi P_{0}$. This finding confirms the results of several previous studies, reporting that $\varphi P_{0}$ is relatively insensitive to decreased leaf water contents (e.g., $[23,24]$ ).

As in the case of $\mathrm{PI}_{\mathrm{abs}}$, the change of $\varphi P_{0}$ was highly similar for HS and CS plants. The decrease of $\varphi P_{0}$ was mainly caused by a decrease of $F_{M}$ and not by an increase of $F_{0}$ (data not shown), indicating that leaf temperature did not exceed the critical temperature for PSII [25], which is associated with a steep increase of $F_{0}$. Interestingly, $\mathrm{PI}_{\text {abs }}$ and $\varphi P_{0}$ only recovered (partially) in the case of CS, while HS plants did not show increased values after 24 hours under standard growing conditions. 


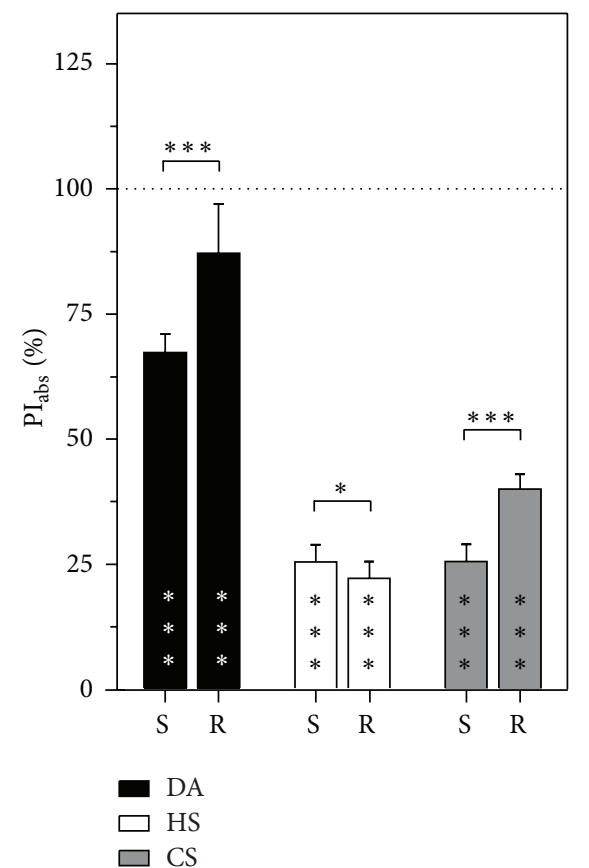

(a)

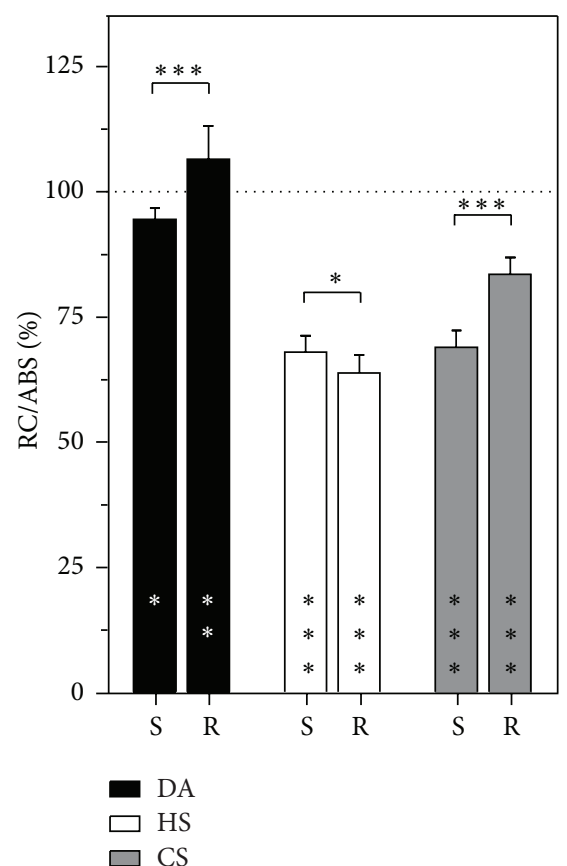

(c)

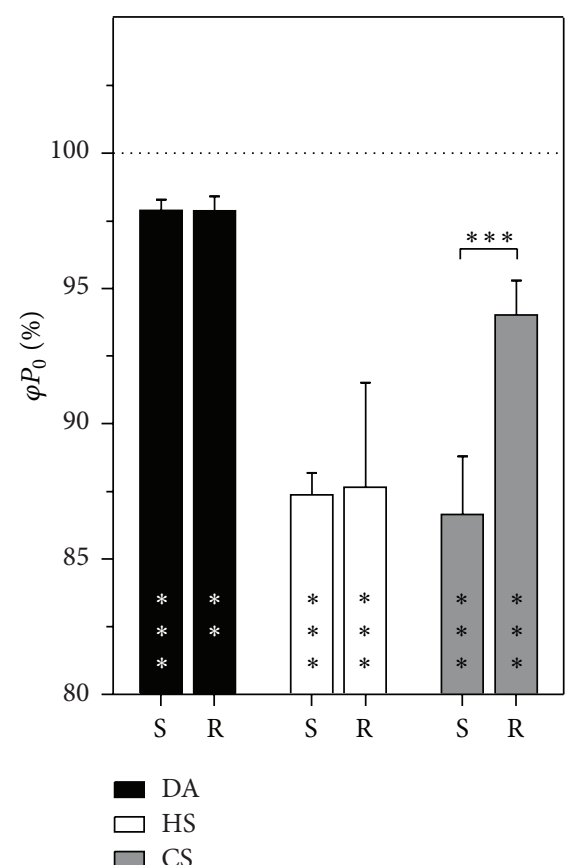

(b)

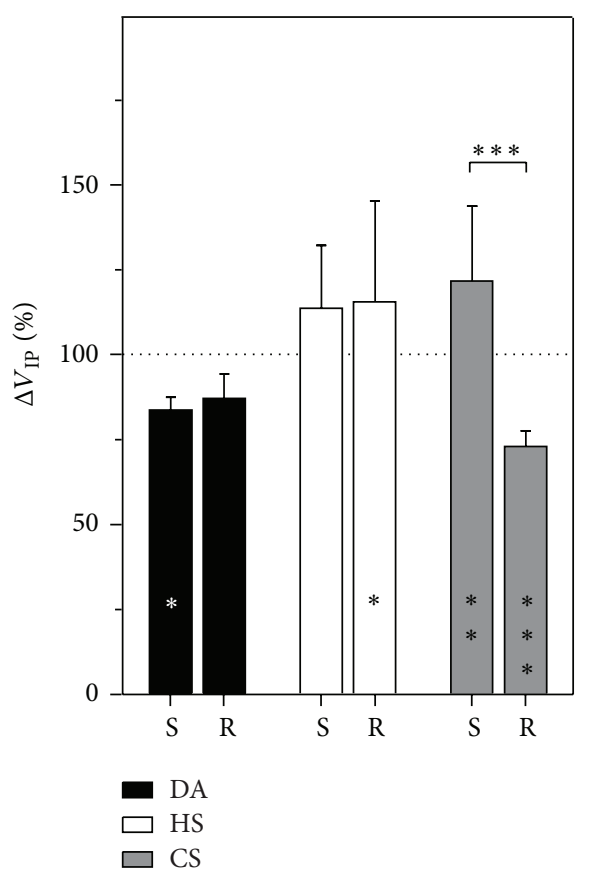

(d)

Figure 1: Effect of drought, heat, or their combination on Performance Index ( $\left.\mathrm{PI}_{\mathrm{abs}}, \mathrm{a}\right)$, the maximum quantum yield of PSII $\left(\varphi P_{0}\right.$, b), the relative fraction of active reaction centers $(\mathrm{RCs} / \mathrm{ABS}, \mathrm{c})$, and the relative amplitude of the IP-phase of the OJIP induction curve $\left(\Delta V_{\text {IP }}, \mathrm{d}\right)$. Values are presented relative to the unstressed control group; DA = drought acclimated, HS = heat stress, $C S=$ combined stresses, $S=$ stress, and $\mathrm{R}=$ recovery; mean $+\mathrm{SD}, n=10$. Asterisks inside the columns represent significance in comparison to control values (=100\%), asterisks above the connecting brackets indicate significance between stress and recovery within one treatment. Significant changes were marked with one $(p<0.05)$, two $(p<0.01)$, or three $(p<0.001)$ asterisks. 


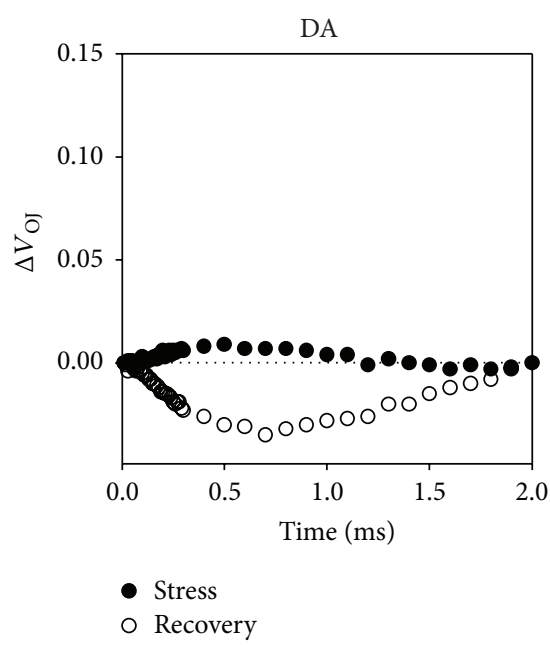

(a)

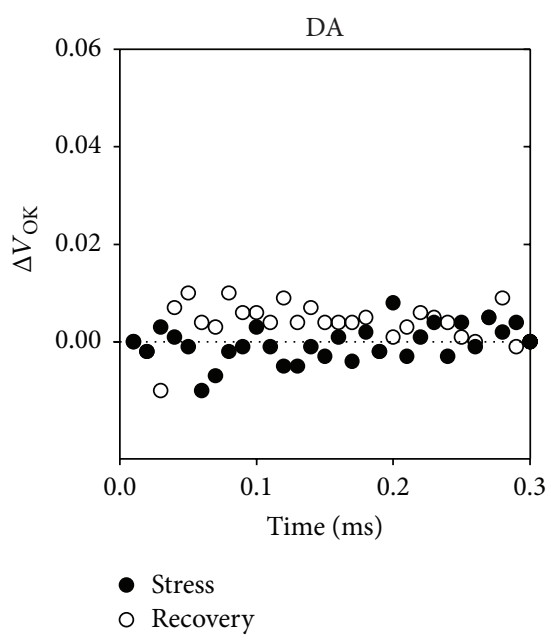

(d)

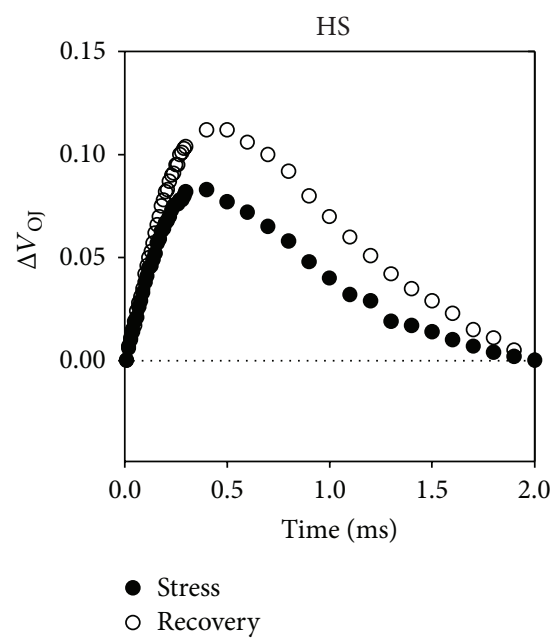

(b)

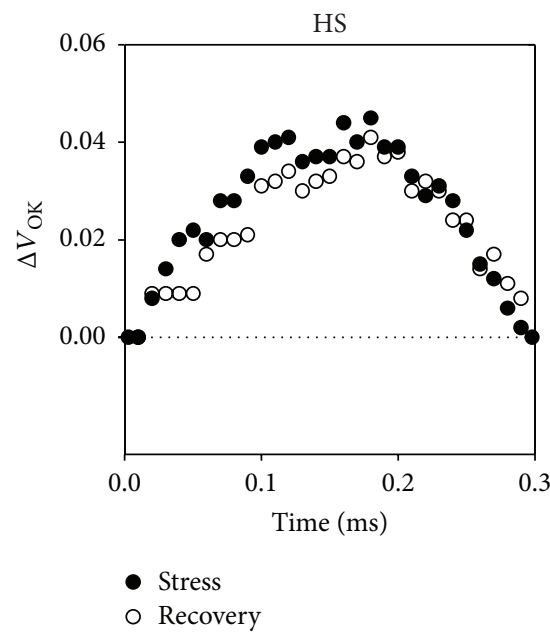

(e)

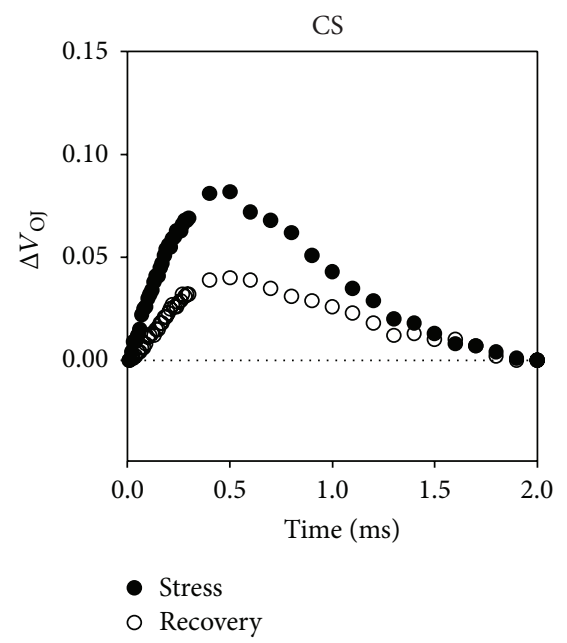

(c)

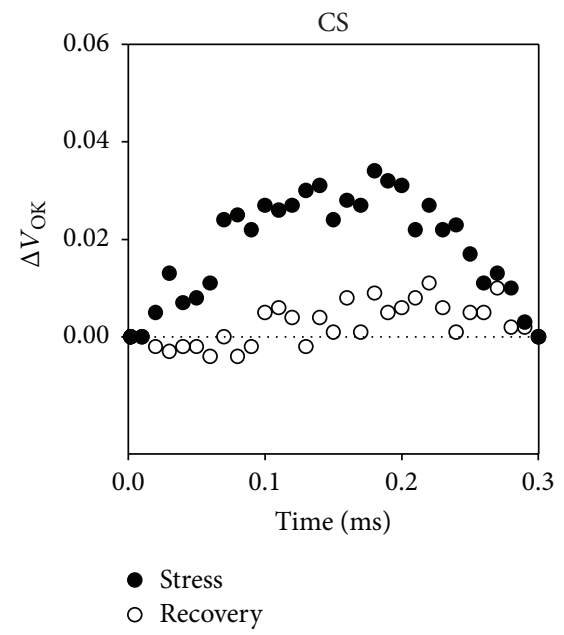

(f)

FIGURE 2: Differences of the relative variable fluorescence of O-J-normalised $\left(\Delta V_{\mathrm{OJ}}\right.$, a-c) and O-K-normalised $\left(\Delta V_{\mathrm{OK}}, \mathrm{d}-\mathrm{f}\right)$ induction curves of wild barley plants suffering drought, heat, or their combination and after 24 hours of recovery: $\Delta V_{\mathrm{OJ}}(t)=\left[\left(F_{t}-F_{0}\right)_{\mathrm{DA} / \mathrm{HS} / \mathrm{CS}} /\left(F_{\mathrm{J}}-F_{0}\right)_{\mathrm{DA} / \mathrm{HS} / \mathrm{CS}}\right]-$ $\left[\left(F_{t}-F_{0}\right)_{\mathrm{CTR}} /\left(F_{\mathrm{J}}-F_{0}\right)_{\mathrm{CTR}}\right], \Delta V_{\mathrm{OK}}(t)=\left[\left(F_{t}-F_{0}\right)_{\mathrm{DA} / \mathrm{HS} / \mathrm{CS}} /\left(F_{\mathrm{K}}-F_{0}\right)_{\mathrm{DA} / \mathrm{HS} / \mathrm{CS}}\right]-\left[\left(F_{t}-F_{0}\right)_{\mathrm{CTR}} /\left(F_{\mathrm{J}}-F_{0}\right)_{\mathrm{CTR}}\right]$; CTR = control, DA $=$ drought acclimated, $\mathrm{HS}=$ heat stress, and $\mathrm{CS}=$ combined stresses; mean, $n=10$.

HS and CS reduced the fraction of $Q_{A}$-reducing RCs. Non- $Q_{A}$-reducing $R C s$ are thought to act as "quenching sinks," dissipating the absorbed light energy exclusively as heat [15]. A reduced RC/ABS was already observed in other HS studies for soybean [26] and for wild barley [27]. Increased heat dissipation under environmental stress can be interpreted as a protective mechanism, as it reduces the risk of overexcitation of PSII under conditions of diminished linear electron transport. Like for the parameters $\mathrm{PI}_{\mathrm{abs}}$ and $\varphi P_{0}, \mathrm{RC} / \mathrm{ABS}$ partially recovered in CS plants while there was no recovery of active RCs in HS plants.

Oukarroum et al. [28] reported for varieties of cultivated barley that the IP-phase of the OJIP transient is sensitive to drought treatment. Reduced $\Delta V_{\mathrm{IP}}$ under drought stress was correlated with a decrease of the relative $\mathrm{PI}_{\mathrm{abs}}$, expressed as the so-called drought factor index (DFI). Additionally, it was shown for wild barley under progressive drought that during the early stages of drought stress the differential relative variable fluorescence around the I-step reacts more sensitively than the preceding J-step [18]. Our results for DA plants confirm the sensitivity of $\Delta V_{\text {IP }}$ and show that a recovery period of $24 \mathrm{~h}$ is not sufficient for restoring the initial kinetics of the IP-phase. The amplitude of IP-phase was assigned to be caused by a reduction of PSI content by correlating $\Delta V_{\text {IP }}$ with the relative amount of the PSI psaD protein as well as with the maximum amplitude of $820 \mathrm{~nm}$ transmission [29]. Accordingly, the authors suggested the use of $\Delta V_{\mathrm{IP}}$ as a semiquantitative measure of changes of the PSI content.

While DA decreased the amplitude of the IP-phase, HS and CS treatment increased $\Delta V_{\mathrm{IP}}$. Since $\Delta V_{\mathrm{IP}}$ is associated with the ratio of functional PSI to functional PSII units rather than with the absolute content of PSI [29], this is not to be interpreted as an increase in PSI units. It is rather to be seen 


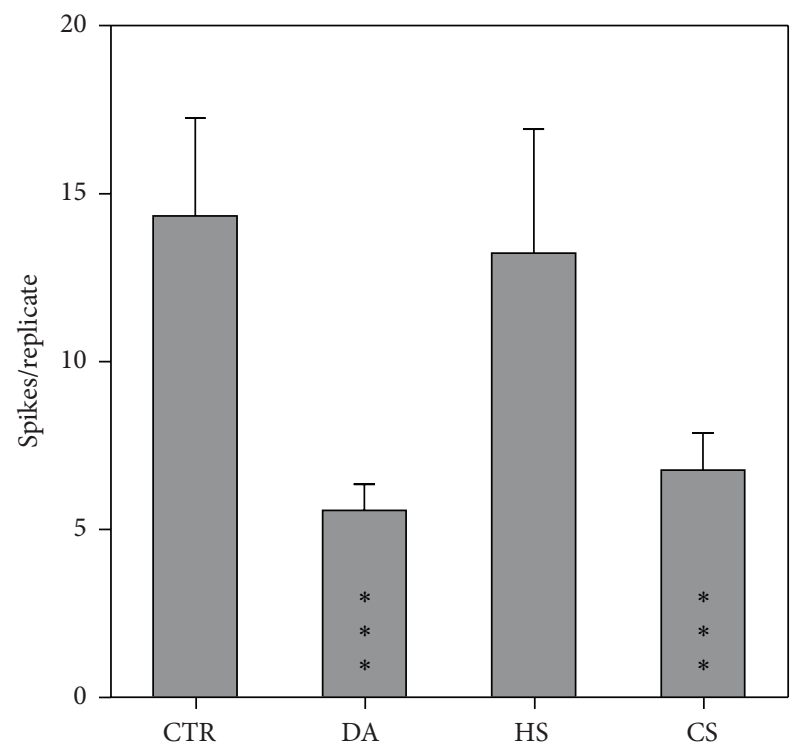

(a)

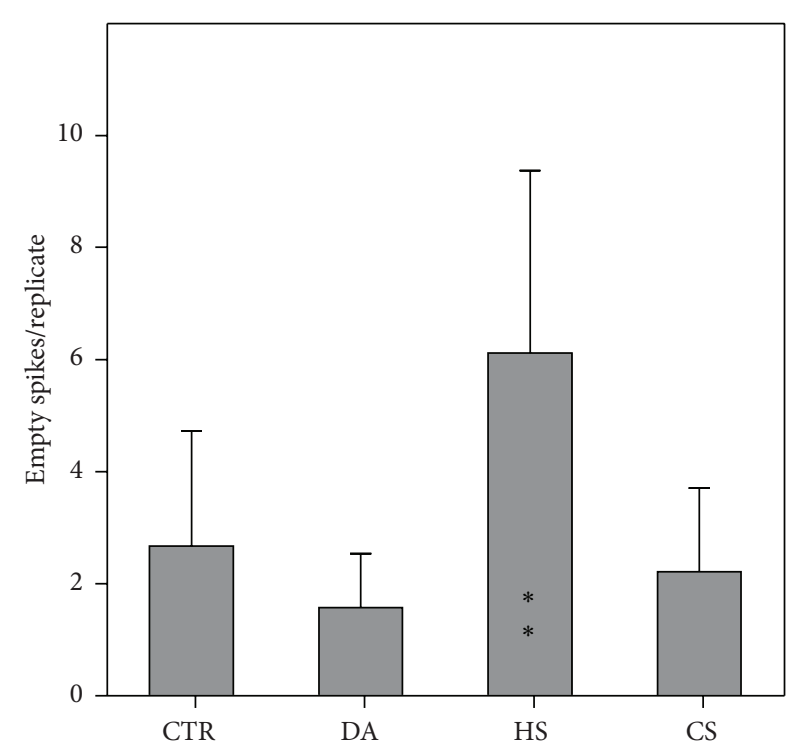

(b)

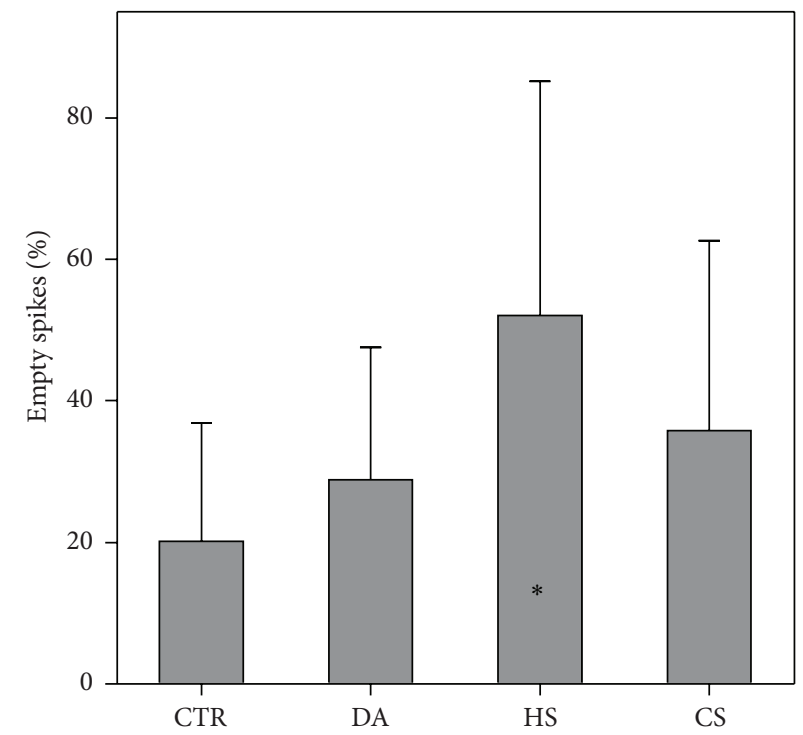

(c)

Figure 3: Number of spikes (a), number of empty spikes (b), and relative fraction of empty spikes (c) under control conditions and after drought acclimation, heat, or combined stresses treatment; CTR = control, DA = drought acclimated, HS = heat stress, and CS = combined stresses; mean $+\mathrm{SD}, n=10$. Asterisks inside the columns represent significance in comparison to control values. Significant changes were marked with one $(p<0.05)$, two $(p<0.01)$, or three $(p<0.001)$ asterisks.

as the result of the higher tolerance of PSI over PSII to HS [30]. Zushi et al. [31] confirmed this finding using the JIPtest analysis for tomato leaves under HS. CS plants showed the same behavior as HS plants immediately after the HS. However, after recovery, PSII functionality has been restored largely (cf. Figure 1(b)), and now the (persisting) effect of the DA on the PSI to PSII ratio became evident.

The fluorescence rise during the first $2 \mathrm{~ms}$ in the normalised OJIP induction curve offers additional information on developing limitations on the donor side of PSII, when visualized in the $\Delta V_{\mathrm{OI}}$ curves. HS and CS initiated the appearance of a so-called K-band at $300-400 \mu$ s (Figures 2(b) and 2(c)). The K-band is a characteristic feature of heat induced damage of PSII structure and was observed in several studies [17, 32]. While, in principle, changes in the earliest phase of the fluorescence induction curve can also be due to differences in antenna size or in the plastoquinone pool redox state, the general interpretation of such effects under heat stress is to attribute it to damage to the electron donor side of PSII, for example, by structural damage of the OEC $[33,34]$. Under DA treatment, no K-band developed (Figure 2(a)), in contrast to observations of Oukarroum et al. [22] and Jedmowski et al. [21] for barley under severe drought stress. An increased relative fluorescence in the OK-phase $\left(\Delta V_{\mathrm{OK}}\right.$, 


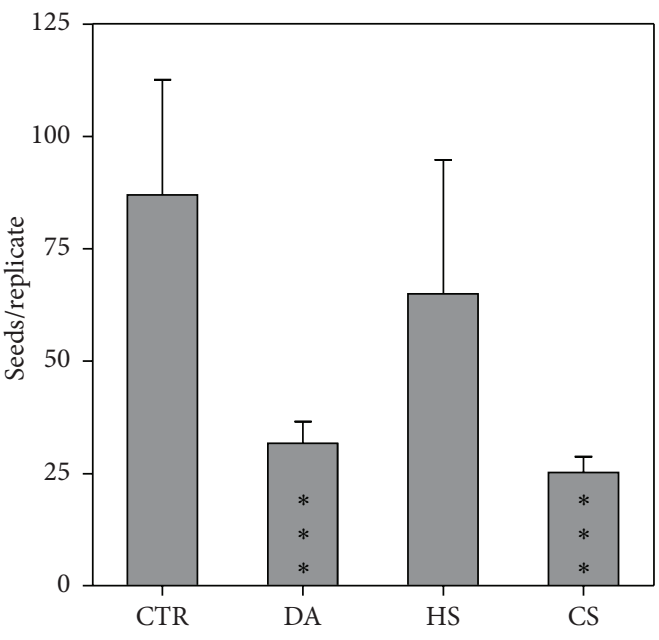

(a)

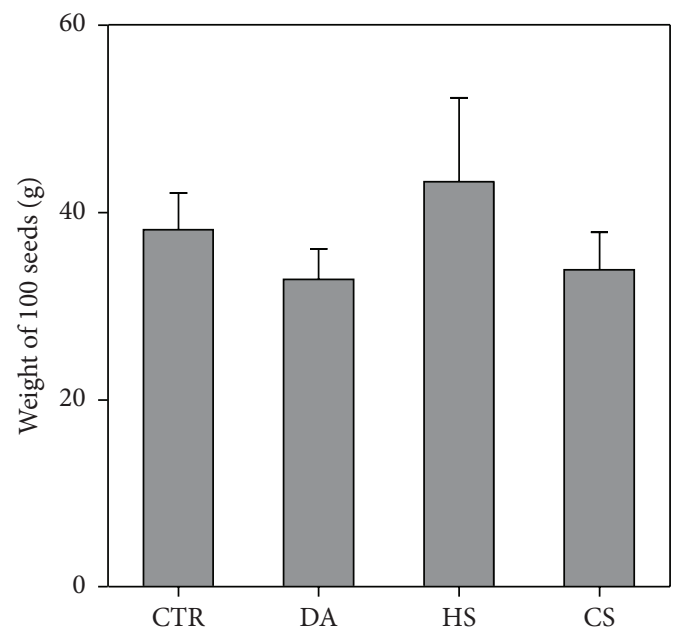

(b)

FIGURE 4: Number of seeds per replicate (a) and weight of 1000 seeds (b) under control conditions and after drought acclimation, heat, or combined stresses treatment; CTR $=$ control, $\mathrm{DA}=$ drought acclimated, HS $=$ heat stress, and CS $=$ combined stresses; mean $+\mathrm{SD}, n=10$. Asterisks inside the columns represent significance in comparison to control values. Significant changes were marked with one $(p<0.05)$, two $(p<0.01)$, or three $(p<0.001)$ asterisks.

"L-band") developed under HS and CS conditions, which was absent under DA treatment. The appearance of the Lband is thought to be associated with decreased energetic connectivity of the PSII units [35], leading to fluorescence emission from those antenna chlorophylls, which are unable to transfer their excitation energy into the RC. While the $\mathrm{K}$ - and L-band under CS treatment were largely reversible (Figures 2(c) and 2(f)), the effect of HS alone pertained over 24 hours (Figures 2(b) and 2(e)).

The persistence of the peaks in the $\Delta V_{\mathrm{OJ}}$ and $\Delta V_{\mathrm{OK}}$ curves in case of the HS plants is remarkable. The recovery processes of PSII after a heat pulse treatment $\left(20-40 \mathrm{~s}\right.$ at $\left.50^{\circ} \mathrm{C}\right)$ were studied by Tóth et al. [36] using barley seedlings. Here, a K-band developed after heat treatment and disappeared entirely after $4 \mathrm{~h}$ of recovery. The authors proposed a lightdependent mechanism including the breakdown of damaged PSII RCs after 4 hours and the de novo protein synthesis of PSII units within $24 \mathrm{~h}$, leading to restoral of the initial OJIP kinetics. It is difficult to judge why our plants did not show the same behavior, as they had a light period during the 24 hours of recovery. In difference to Tóth et al. [36], we did not use a short heat pulse, but a longer treatment at a lower temperature, including an acclimation phase (gradual increase of temperature). In consequence, during our treatment, plants had the possibility to adjust and regulate their metabolism and gene expression, for example, by the production of heat shock proteins [37]. It might be speculated that under our experimental conditions different mechanisms of regulation of PSII induced by the acclimation to high temperatures may be responsible for the apparently different results. The results of the chlorophyll fluorescence measurements clearly suggest the induction of (a) protective mechanism(s) during DA, resulting in a better PSII recovery of CS plants from heat treatment in comparison to the HS group, while the acute effect of heat was highly similar for HS and CS plants.

4.2. Agronomic Measurements. The effect of the stresses on photosynthesis and growth processes is best integrated in the final yield of the plants, given as number of seeds per replicate and seed weight (Figures 4(a) and 4(b)). The continuous and moderate drought treatment resulted in a significant decrease of the number of spikes and seeds per replicate as a consequence of the continuously lower overall carbon assimilation. The same effect was also found under CS, most likely as a result of the drought component. HS, in contrast, affected the production of fertile spikes significantly; however, in DA plants, this was not the case. In wheat plants, increasing temperatures above $30^{\circ} \mathrm{C}$ during the transition from the vegetative to the reproductive stage and during floret formation resulted in complete sterility [38]. Additionally, floral abnormalities induced by HS lead to spikelet sterility in rice production [39]. In principle, there are two possible explanations for the smaller proportion of empty spikes in the CS group. Firstly, CS plants may have induced a protection mechanism during DA treatment, leading to a "crossresistance" of floral development to increased temperatures. Secondly, the DA treatment may have shifted the onset or the speed of flowering. If this would be the case, the applied heat treatment would have affected CS and HS plants at different stages of floral development. Guan et al. [40] described an acceleration of heading for rice cultivars under drought stress, which can be interpreted as a drought escape strategy ensuring flower development before a critical degree of plant desiccation is reached. On the other hand, Rollins et al. [41] did not observe any effect of drought stress in two cultivated barley genotypes concerning the time to reach maturity. Since the number of spikes per replicate was the same for DA and 
CS plants (and lower than for CTR plants), we suggest that this feature was determined before the heat treatment. On the other hand, seed weight was not significantly affected by any of the stresses. Rollins et al. [41] found long-term HS (one week at $36^{\circ} / 32^{\circ} \mathrm{C}$ day/night temperatures) and CS to significantly affect seed weight, while drought alone had no effect on this parameter in the case of $H$. vulgare. We conclude that the drought component of our CS treatment dominated the (short-term) heat component in our experiment, and consequently the seed weight was not affected in our case.

\section{Concluding Remarks and Outlook}

Measurements of fast chlorophyll fluorescence induction curves revealed a characteristic pattern of inhibitions of the photosynthetic electron transport. In the case of longterm drought treatment, fluorescence data could be used for estimating productivity, as the reduced yield was directly related to photosynthetic limitations. For HS, disturbances of floral development were the decisive feature determining productivity. In this case, chlorophyll fluorescence data is uncoupled from agronomic traits. This is also obvious for plants suffering CS, as their advantage over well-watered plants in restoring the photosynthetic electron transport after heat stress had no impact on productivity; that is, their grain yield was irreversibly determined during the shortterm heat treatment. However, under conditions of a longterm high growth temperature, as applied, for example, in the experiments performed by Rollins et al. [41], a negative impact of heat on photosynthesis may again lead to a coupling of fluorescence parameters and agronomic traits. This should be clarified in future experiments. In summary, we conclude that chlorophyll fluorescence is a useful tool assisting in estimating crop performance under environmental stresses, though its meaningful use is restricted to situations in which carbon fixation is the dominating cause of yield limitation.

\section{Abbreviations}

$\begin{array}{ll}\text { CS: } & \text { Combined stresses } \\ \text { CTR: } & \text { Control } \\ \text { DA: } & \text { Drought acclimation } \\ \text { HS: } & \text { Heat stress } \\ \text { OEC: } & \text { Oxygen evolving complex } \\ \text { PI }_{\text {abs }}: & \text { Performance Index on absorption basis } \\ \text { PSII: } & \text { Photosystem II } \\ \text { RC: } & \text { Reaction center } \\ \text { Rubisco: } & \text { Ribulose 1,5-bisphosphate } \\ & \text { carboxylase/oxygenase } \\ \text { RWC: } & \text { Relative leaf water content } \\ \text { SWC: } & \text { Volumetric soil water content } \\ \Delta V_{\text {IP }}: & \text { Relative amplitude of the IP-phase of the } \\ & \text { OJIP induction curve } \\ \Psi: & \text { Soil water potential. }\end{array}$

\section{Conflict of Interests}

The authors declare that there is no conflict of interests regarding the publication of this paper.

\section{Acknowledgments}

This study was funded by the German Federal Ministry of Education and Research, Project no. 01DH14007, and the Science and Technology Development Fund, Egypt, Project no. 5034, in the framework of the German Egyptian Research Fund (GERF).

\section{References}

[1] D. S. Battisti and R. L. Naylor, "Historical warnings of future food insecurity with unprecedented seasonal heat," Science, vol. 323, no. 5911, pp. 240-244, 2009.

[2] G. C. Nelson, D. van der Mensbrugghe, H. Ahammad et al., "Agriculture and climate change in global scenarios: why don't the models agree," Agricultural Economics, vol. 45, no. 1, pp. 85101, 2014.

[3] IPCC, "Summary for policymakers", in Climate Change 2013: The Physical Science Basis. Contribution of Working Group I to the Fifth Assessment Report of the Intergovernmental Panel on Climate Change, T. F. Stocker, D. Qin, G. K. Plattner et al., Eds., Cambridge University Press, Cambridge, UK, 2013.

[4] J. R. Harlan and D. Zohary, "Distribution of wild wheats and barley," Science, vol. 153, no. 3740, pp. 1074-1080, 1966.

[5] T.-M. Choo, "Genetic resources of tibetan barley in China," Crop Science, vol. 42, no. 5, pp. 1759-1760, 2002.

[6] R. P. Ellis, B. P. Forster, L. L. Handley et al., "Wild barley: a source of genes for crop improvement in the 21st century?" Journal of Experimental Botany, vol. 51, no. 342, pp. 9-17, 2000.

[7] N. Suzuki, R. M. Rivero, V. Shulaev, E. Blumwald, and R. Mittler, "Abiotic and biotic stress combinations," New Phytologist, vol. 203, no. 1, pp. 32-43, 2014.

[8] P. V. V. Prasad, S. R. Pisipati, I. Momčilović, and Z. Ristic, "Independent and combined effects of high temperature and drought stress during grain filling on plant yield and chloroplast EF-Tu expression in spring wheat," Journal of Agronomy and Crop Science, vol. 197, no. 6, pp. 430-441, 2011.

[9] D. W. Lawlor and W. Tezara, "Causes of decreased photosynthetic rate and metabolic capacity in water-deficient leaf cells: a critical evaluation of mechanisms and integration of processes," Annals of Botany, vol. 103, no. 4, pp. 561-579, 2009.

[10] U. Feller, S. J. Crafts-Brandner, and M. E. Salvucci, "Moderately high temperatures inhibit ribulose-1,5-bisphosphate carboxylase/oxygenase (Rubisco) activase-mediated activation of Rubisco," Plant Physiology, vol. 116, no. 2, pp. 539-546, 1998.

[11] S. J. Crafts-Brandner and M. E. Salvucci, "Rubisco activase constrains the photosynthetic potential of leaves at high temperature and $\mathrm{CO}_{2}$," Proceedings of the National Academy of Sciences of the United States of America, vol. 97, no. 24, pp. 13430-13435, 2000.

[12] D. Nash, M. Miyao, and N. Murata, "Heat inactivation of oxygen evolution in Photosystem II particles and its acceleration by chloride depletion and exogenous manganese," Biochimica et Biophysica Acta, vol. 807, no. 2, pp. 127-133, 1985.

[13] M. Brestič and M. Živčák, "PSII fluorescence techniques for measurement of drought and high temperature stress signal in crop plants: protocols and applications," in Molecular Stress Physiology of Plants, G. R. Rout and A. B. Das, Eds., pp. 87-131, Springer, New Delhi, India, 2013.

[14] R. J. Strasser, A. Srivastava, and M. Tsimilli-Michael, "The fluorescence transient as a tool to characterize and screen photosynthetic samples," in Probing Photosynthesis: Mechanisms, Regulation and Adaption, M. Yunus, U. Pathre, and P. Mohanty, Eds., pp. 445-483, Taylor \& Francis, London, UK, 2000. 
[15] R. J. Strasser, M. Tsimilli-Michael, and A. Srivastava, "Analysis of the chlorophyll a fluorescence transient," in Chlorophyll a Fluorescence: A Signature of Photosynthesis, G. C. Papageorgiou and Govindjee, Eds., vol. 19 of Advances in Photosynthesis and Respiration, pp. 321-362, Springer, Dordrecht, The Netherlands, 2004.

[16] A. J. Strauss, G. H. J. Krüger, R. J. Strasser, and P. D. R. van Heerden, "Ranking of dark chilling tolerance in soybean genotypes probed by the chlorophyll a fluorescence transient O-J-I-P," Environmental and Experimental Botany, vol. 56, no. 2, pp. 147-157, 2006.

[17] S. Mathur, A. Jajoo, P. Mehta, and S. Bharti, "Analysis of elevated temperature-induced inhibition of photosystem II using chlorophyll a fluorescence induction kinetics in wheat leaves (Triticum aestivum)," Plant Biology, vol. 13, no. 1, pp. 16, 2011.

[18] C. Jedmowski, S. Bayramov, and W. Brüggemann, "Comparative analysis of drought stress effects on photosynthesis of Eurasian and North African genotypes of wild barley," Photosynthetica, vol. 52, no. 4, pp. 564-573, 2014.

[19] H. Lambers, F. S. Chapin III, and T. L. Pons, "Assumptions and approaches," in Plant Physiological Ecology, H. Lambers, F. S. Chapin, and T. L. Pons, Eds., pp. 1-9, Springer, New York, NY, USA, 1998.

[20] R. E. Smart and G. E. Bingham, "Rapid estimates of relative water content," Plant Physiology, vol. 53, no. 2, pp. 258-260, 1974.

[21] C. Jedmowski, A. Ashoub, and W. Brüggemann, "Reactions of Egyptian landraces of Hordeum vulgare and Sorghum bicolor to drought stress, evaluated by the OJIP fluorescence transient analysis," Acta Physiologiae Plantarum, vol. 35, no. 2, pp. 345354, 2013.

[22] A. Oukarroum, S. E. Madidi, G. Schansker, and R. J. Strasser, "Probing the responses of barley cultivars (Hordeum vulgare L.) by chlorophyll a fluorescence OLKJIP under drought stress and re-watering," Environmental and Experimental Botany, vol. 60, no. 3, pp. 438-446, 2007.

[23] D. Christen, S. Schönmann, M. Jermini, R. J. Strasser, and G. Défago, "Characterization and early detection of grapevine (Vitis vinifera) stress responses to esca disease by in situ chlorophyll fluorescence and comparison with drought stress," Environmental and Experimental Botany, vol. 60, no. 3, pp. 504514, 2007.

[24] M. Živčák, M. Brestič, K. Olšovská, and P. Slámka, "Performance index as a sensitive indicator of water stress in Triticum aestivum L," Plant, Soil and Environment, vol. 54, no. 4, pp. 133139, 2008.

[25] U. Schreiber and J. A. Berry, "Heat-induced changes of chlorophyll fluorescence in intact leaves correlated with damage of the photosynthetic apparatus," Planta, vol. 136, no. 3, pp. 233-238, 1977.

[26] J. A. De Ronde, W. A. Cress, G. H. J. Krüger, R. J. Strasser, and J. Van Staden, "Photosynthetic response of transgenic soybean plants, containing an Arabidopsis P5CR gene, during heat and drought stress," Journal of Plant Physiology, vol. 161, no. 11, pp. 1211-1224, 2004.

[27] C. Jedmowski and W. Brüggemann, "Imaging of fast chlorophyll fluorescence induction curve (OJIP) parameters, applied in a screening study with wild barley (Hordeum spontaneum) genotypes under heat stress," Journal of Photochemistry and Photobiology B: Biology, vol. 151, pp. 153-160, 2015.

[28] A. Oukarroum, G. Schansker, and R. J. Strasser, "Drought stress effects on photosystem i content and photosystem II thermotolerance analyzed using $\mathrm{Chl}$ a fluorescence kinetics in barley varieties differing in their drought tolerance," Physiologia Plantarum, vol. 137, no. 2, pp. 188-189, 2009.

[29] M. G. Ceppi, A. Oukarroum, N. Çiçek, R. J. Strasser, and G. Schansker, "The IP amplitude of the fluorescence rise OJIP is sensitive to changes in the photosystem I content of leaves: a study on plants exposed to magnesium and sulfate deficiencies, drought stress and salt stress," Physiologia Plantarum, vol. 144, no. 3, pp. 277-288, 2012.

[30] M. Havaux, H. Greppin, and R. J. Strasser, "Functioning of photosystems I and II in pea leaves exposed to heat stress in the presence or absence of light. Analysis using in-vivo fluorescence, absorbance, oxygen and photoacoustic measurements," Planta, vol. 186, no. 1, pp. 88-98, 1991.

[31] K. Zushi, S. Kajiwara, and N. Matsuzoe, "Chlorophyll a fluorescence OJIP transient as a tool to characterize and evaluate response to heat and chilling stress in tomato leaf and fruit," Scientia Horticulturae, vol. 148, pp. 39-46, 2012.

[32] A. Oukarroum, S. El Madidi, and R. J. Strasser, "Exogenous glycine betaine and proline play a protective role in heatstressed barley leaves (Hordeum vulgare L.): A chlorophyll a fluorescence study," Plant Biosystems, vol. 146, no. 4, pp. 10371043, 2012.

[33] S. Z. Tóth, G. Schansker, G. Garab, and R. J. Strasser, "Photosynthetic electron transport activity in heat-treated barley leaves: the role of internal alternative electron donors to photosystem II," Biochimica et Biophysica Acta, vol. 1767, no. 4, pp. 295-305, 2007.

[34] G. P. Wang, F. Li, J. Zhang, M. R. Zhao, Z. Hui, and W. Wang, "Overaccumulation of glycine betaine enhances tolerance of the photosynthetic apparatus to drought and heat stress in wheat," Photosynthetica, vol. 48, no. 1, pp. 30-41, 2010.

[35] R. J. Strasser and A. D. Stirbet, "Estimation of the energetic connectivity of PS II centres in plants using the fluorescence rise O-J-I-P: fitting of experimental data to three different PS II models," Mathematics and Computers in Simulation, vol. 56, no. 4-5, pp. 451-461, 2001.

[36] S. Z. Tóth, G. Schansker, J. Kissimon, L. Kovács, G. Garab, and R. J. Strasser, "Biophysical studies of photosystem IIrelated recovery processes after a heat pulse in barley seedlings (Hordeum vulgare L.)," Journal of Plant Physiology, vol. 162, no. 2, pp. 181-194, 2005.

[37] A. Ashoub, M. Baeumlisberger, M. Neupaertl, M. Karas, and W. Brüggemann, "Characterization of common and distinctive adjustments of wild barley leaf proteome under drought acclimation, heat stress and their combination," Plant Molecular Biology, vol. 87, no. 4-5, pp. 459-471, 2015.

[38] H. S. Saini and D. Aspinall, "Abnormal sporogenesis in wheat (Triticum aestivum L.) induced by short periods of high temperature," Annals of Botany, vol. 49, no. 6, pp. 835-846, 1982.

[39] Y. Takeoka, K. Hiroi, H. Kitano, and T. Wada, "Pistil hyperplasia in rice spikelets as affected by heat stress," Sexual Plant Reproduction, vol. 4, no. 1, pp. 39-43, 1991.

[40] Y. S. Guan, R. Serraj, S. H. Liu et al., "Simultaneously improving yield under drought stress and non-stress conditions: a case study of rice (Oryza sativa L.)," Journal of Experimental Botany, vol. 61, no. 15, pp. 4145-4156, 2010.

[41] J. A. Rollins, E. Habte, S. E. Templer, T. Colby, J. Schmidt, and M. von Korff, "Leaf proteome alterations in the context of physiological and morphological responses to drought and heat stress in barley (Hordeum vulgare L.)," Journal of Experimental Botany, vol. 64, no. 11, pp. 3201-3212, 2013. 

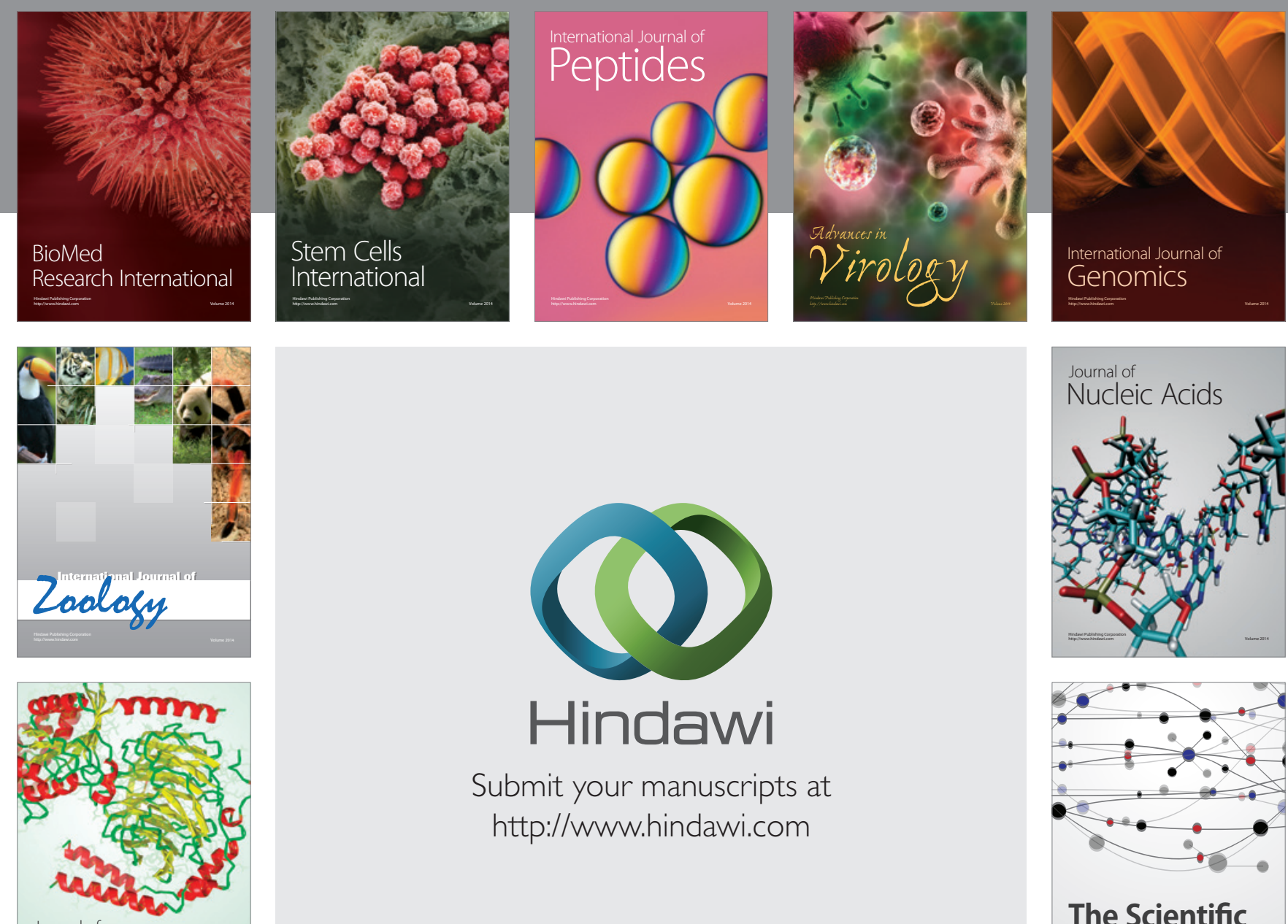

Submit your manuscripts at

http://www.hindawi.com

Journal of
Signal Transduction
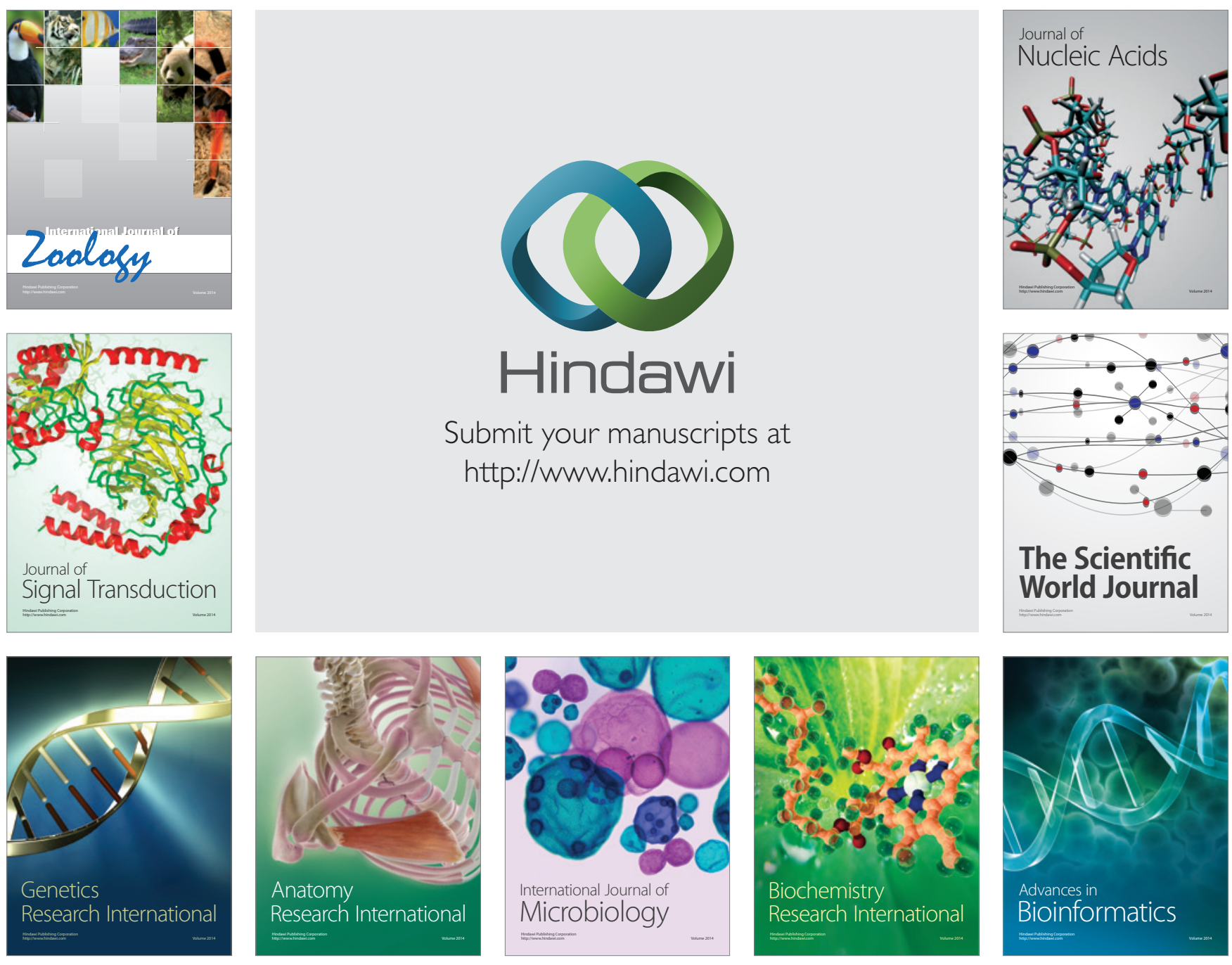

The Scientific World Journal
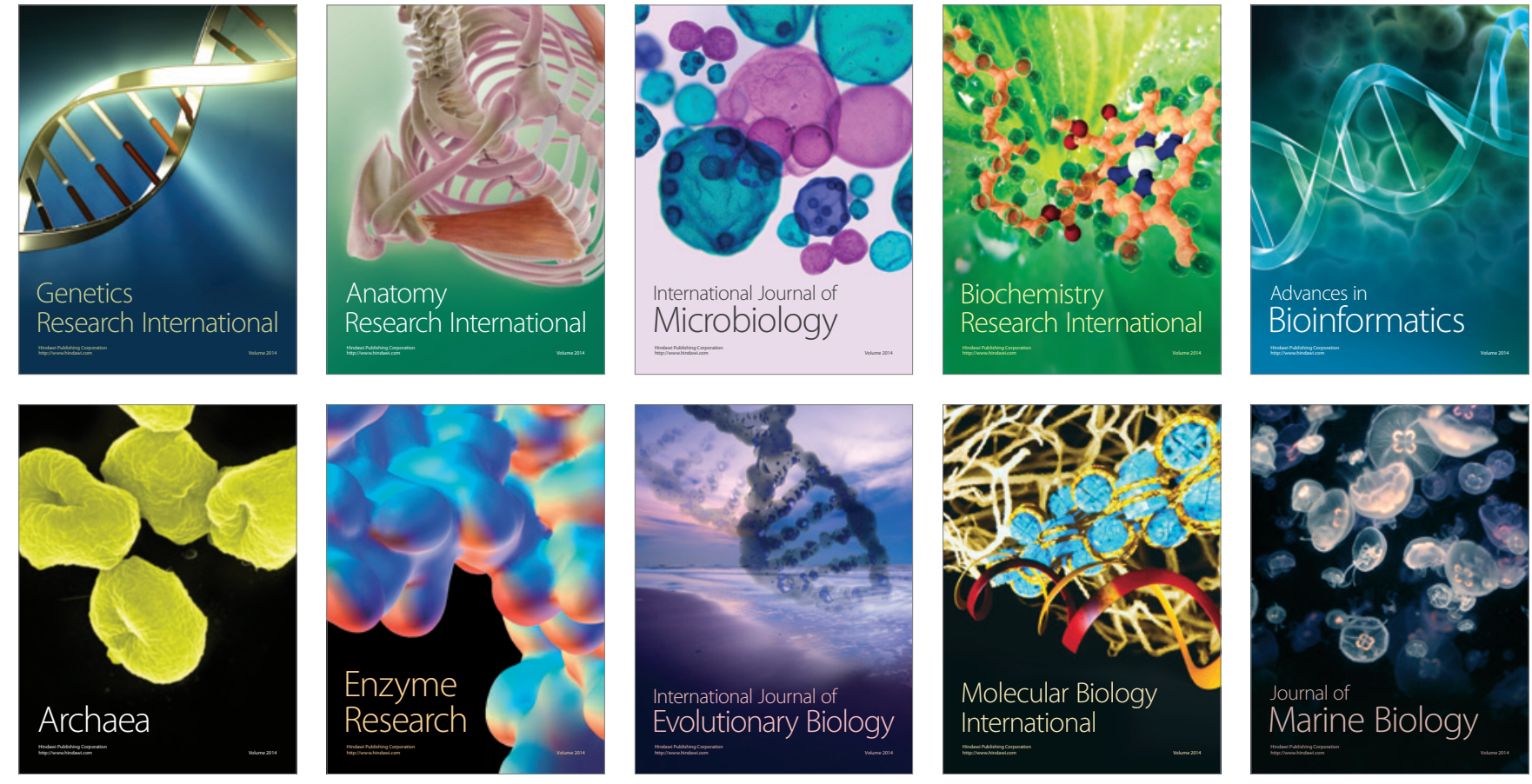